\title{
RELATION BETWEEN THE SHAPLEY VALUE AND NULL GROUP COHOMOLOGY
}

\author{
AndRzeJ Madrecki
}

\begin{abstract}
The purpose of this paper is to give an application of homological methods to game theory. The main theorems give conditions of a homological nature for the existence and uniqueness of the Shapley value for games with continuum players.
\end{abstract}

\section{Introduction}

The Shapley value for $n$-person games was introduced in [3]. In [1] Aumann and Shapley have given the following definition of the Shapley value for games of continuum players: Let $I=[0,1]$ be the set of players, let $B V$ denote the space of all bounded variation set functions defined on Borel sets $B$ of $I$, and let $F A$ denote the set of all bounded finitely additive set functions on $(I, B)$. Finally, let $G$ be the group of all Borel automorphisms of the interval. $I$. Then a Shapley value is a map $\varphi: Q \mapsto F A$, where $Q$ is a G-invariant subspace of $B V$, and $\varphi$ is linear, positive and it satisfies the following conditions:

(I) $\varphi g_{*}=g_{*} \varphi$ for all $g \in G$;

(II) $(\varphi v)(I)=v(I)$ for all $v \in Q$. (Here $\left(g_{\star} \varphi\right)(S):=\varphi(g(S))$ for $g \in G$ and $S \in B$.)

In [1] there were investigated the subspaces $Q$ of $B V$ for which

Received 22 June 1983.

Copyright Clearance Centre, Inc. Serial-fee code: 0004-9727/83 $\$ A 2.00+0.00$. 
there exists the Shapley value $\varphi$ and also those for which $\varphi$ is uniquely determined. A natural question arises: how many Shapley values exist for a given linear space and how it depends on the group $G$. In this paper we give a partial answer to this question using the homological methods.

Since axioms (I) and (II) state a functor property in the category of $G$-modules, it apparently leads to the algebraic approach to the problem of existence and uniqueness of the Shapley value.

In Section 1 we define the action of a group $G$ on the Banach algebra $B V$, where $G$ is a group of the Borel automorphisms of a Polish space; and we introduce special cohomological spaces. Section 3 contains the main result of this paper (Theorem 3.1). If the null cohomological space, $H^{0}(G, F A)$ is nontrivial, then there exist $G$-Shapley value on every subspace $Q \supseteq F A$ with $Q \subseteq B V$.

From the proof of Theorem 3.1 it also follows how many distinct Shapley values are available. A necessary condition for the existence exactly one $G$-Shapley value is established in Theorem 3.2.

For basic notions of homological algebra we refer to [2].

\section{Preliminaries and notations}

Let $(X, B)$ be any measurable space, that is, $B$ is a $\sigma$-algebra of subsets of $X$. By $B V$ we denote the Banach space of all real valued set functions of bounded variation defined on $(X, B)$. By $F A$ (respectively $\mathrm{FA}^{+}$) we denote the subspace of all bounded finitely additive (respectively nonnegative) set functions in $B V$ (see [1]).

By $\operatorname{Aut}(X, B)$ we denote the group of all automorphisms of the space $(X, B)$, where the multiplication is defined as follows:

(I.I) $\left(g_{1} \cdot g_{2}\right)(S):=g_{2}\left(g_{1}(S)\right)$ for all $g_{1}, g_{2} \in \operatorname{Aut}(X, B)$, and $S \in B$.

Let $G$ be any subgroup of $\operatorname{Aut}(X, B)$. The action of the group $G$ on the space $B V$, that is, the map

$$
G \times B V \rightarrow(g, v) \mapsto g \cdot v \in B V
$$

is defined by

(1.3) $(g v)(S)=v(g(S))$ for all $g \in G, v \in B V$ and $S \in B$. 
It is easy to verify that the following hold:

(i) $g\left(\alpha v_{1}+\beta v_{2}\right)=\alpha g v_{1}+\beta g v_{2}$,

(ii) $\left(g_{1} g_{2}\right) v=g_{1}\left(g_{2} v\right)$,

(iii) $e v=v$, where $e$ is the neutral element of $G$,

for each $g, g_{1}, g_{2} \in G, v, v_{1}, v_{2} \in B V$ and $\alpha, \beta \in \mathbb{R}$.

Let $R[G]$ be the group algebra of the group $G$ over real numbers, that is, the elements of $R[G]$ have the form

$$
\sum_{g} r_{g} g, \quad r_{g} \in \mathbb{R}, g \in G \text { and } r_{g}=0
$$

for all but finitely many $g \in G$, and the multiplication is defined by

$$
\left(\sum_{g} r_{g} \cdot g\right)\left(\sum_{h} r_{h} \cdot h\right)=\sum_{g, h}\left(r_{g} \cdot r_{h}\right) \cdot(g \cdot h) \text {. }
$$

Let us define the action of $R[G]$ on $B V$ as follows:

$$
\mathrm{R}[G] \times B V \rightarrow\left(\sum_{g} r_{g} \cdot g, v\right) \mapsto \sum_{g} r_{g}(g v) \in B V .
$$

By (1.4), (1.7) and the definition of $R[G]$, it follows that $B V$ may be regarded as $\mathbf{R}[G]$-module. Moreover, the additive group $\mathbf{R}$ of real numbers also is a $R[G]$-module (it is called trivial $R[G]$-module), when the action of $R[G]$ in $R$ is given by

$$
h \cdot r=r \text { for each } r \in \mathbb{R} \text { and } h \in \mathbb{R}[G] \text {. }
$$

For each subgroup $G$ of $\operatorname{Aut}(X, B)$ we define the sequence $\left\{H^{q}(G, \cdot)\right\}, q=0,1,2,3, \ldots$, of $q$-dimensional cohomology groups by setting

$$
H^{q}(G, A):=\operatorname{Ext}_{\mathbf{R}[G]}^{q}(\mathbf{R}, A)
$$

for any $\mathrm{R}[G]$-module $A$ (see [2]). Clearly, each $H^{q}(G, A)$ is a linear space over $\mathbf{R}$. From the definition it follows that $H^{q}(G, \cdot)$ is the covariant functor from the category $\operatorname{Mod}_{\mathrm{R}}[G]$ of all $\mathrm{R}[G]$-modules to the category Vect $\operatorname{R}_{\mathbf{R}}$ of all vector spaces over $R$. 
We note that $\left\{H^{q}(G, \cdot)\right\}_{q}$ has the following properties:

$$
H^{0}(G, A)=\operatorname{Hom}_{\mathbb{R}[G]}(\mathbb{R}, A) \text { for each } \mathbb{R}[G] \text {-module } A,
$$

(1.11) every short exact sequence $0 \mapsto A \mapsto B \mapsto C \mapsto 0$ of $R[G]$-modules induces the long exact sequence

$$
0 \mapsto H^{0}(G, A) \mapsto H^{0}(G, B) \mapsto H^{0}(G, C) \mapsto H^{1}(G, A) \mapsto H^{\perp}(G, B) \mapsto \ldots
$$

of the vector spaces.

LEMMA 1.1. $H^{0}(G, A)=A^{G}$, where

$$
A^{G}=\{a \in A: g a=a \text {, for every } g \in G\} \text {. }
$$

Proof. If $\varphi \in H^{O}(G, A)=\operatorname{Hom}_{\mathbb{R}[G]}(\mathbb{R}, A)$, then $\varphi$ is a function from $\mathbf{R}$ into $A$ such that $\varphi(r)=r \varphi(1)$ for all $r \in \mathbb{R}$. Moreover, for each $g \in G$ we have

$$
\varphi(1)=\varphi(g \cdot 1)=g \cdot \varphi(1) \text {, hence } \varphi(1) \in A^{G} .
$$

Finally, we observe that the map

$$
\operatorname{Hom}_{\mathbf{R}[G]}(\mathbb{R}, A) \ni \varphi \longmapsto \varphi(1) \in A^{g}
$$

is the isomorphism of the vector spaces and this completes the proof.

\section{Definition of the $G$-Shapley value in the framework of cohomology}

$$
\text { Let }||: B V \mapsto B V \text { denote the set function defined by }
$$

$$
|v|(S)=|v(S)| \text { for every } S \in B, v \in B V .
$$

For every $g \in G$ we denote by $g_{*}$ the linear map from $B V$ to $B V$ defined by formula

$$
g_{*}(v)(S)=v(g(S)) \text { for all } v \in B V, g \in G, S \in B .
$$

Following [1], a $G$-Shapley value $\varphi$ on $Q$ is the R-linear map $\varphi: Q \mapsto F A$ satisfying the following conditions:

(2.3) (i) for every $g \in G, g_{*} \varphi=\varphi g_{*}$ (the symmetry);

(ii) $(\varphi v)(X)=v(X)$ for each $v \in Q$ (the Pareto optimality); 
(iii) $\varphi(|v|)=|\varphi(v)|$ for each $v \in Q$.

The condition (2.1) (i) and linearity of $\varphi$ imply that $\varphi \in \operatorname{Hom}_{\mathbb{R}[G]}(Q, F A)$. Now we define the $\mathbb{R}[G]$-homomorphism $I: B V \mapsto \mathbb{R}$ (where $\mathbf{R}$ is regarded as the trivial $\mathbb{R}[G]$-module), by the formula

$$
I(v)=v(X) \text { for each } v \in B V,
$$

where $(X, B)$ is a given measurable space.

For every $Q \subseteq B V$ we denote by $I_{Q}$ the restriction of $I$ to $Q$. Now we are ready for the cohomological definition of the Shapley value.

DEFINITION 1. We shall say that $\varphi \in \operatorname{Hom}_{\mathbb{R}[G]}(Q, F A)$ is the $G$-Shapley value on the linear $G$-invariant subspace $Q \subseteq B V$ if $\varphi$ satisfies two conditions:

$$
\begin{aligned}
& \text { (SVI) } I_{F A} \circ \varphi=I_{Q} ; \\
& \text { (SVII) } \varphi \circ||=|| \circ \varphi .
\end{aligned}
$$

$I_{Q}, I_{F A}$ and || are mappings defined above, and $\circ$ denotes the composition of the maps.

3. Cohomological conditions for the existence of the G-Shapley

$$
\text { value when } H^{0}(G, E A) \text { is nontrivial. }
$$

Necessary condition for the uniqueness of the $G$-Shapley value

Let $\varphi$ be a $G$-Shapley value on $Q$. For any $q \geq 0$, we denote by $\varphi^{*}, q, I_{Q}^{*, q}$ and $I_{F A}^{*}, q$ the homomorphisms induced by $\varphi, I_{Q}$ and $I_{F A}$, respectively. Then we have the following:

LEMMA 3.1. If $\varphi$ is the G-Shapley value on $Q$ then for each $q \geq 0$ the following diagram

$(\mathrm{SVI})_{\mathrm{q}}$

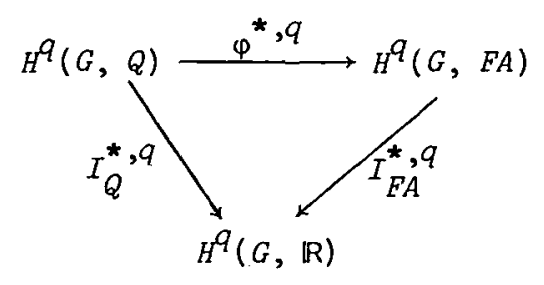

commutes. 
The lemma follows immediately from the cohomological definition of the $G$-Shapley value, and from the functional properties of the cohomology theory .

COROLLARY 3.1. If for some $q \geq 0$ and for each $\mathbb{R}$-linear map $h: H^{q}(G, Q) \mapsto H^{q}(G, F A)$ the diagram (SVI) ${ }_{\mathrm{q}}$ does not commute, then there is no G-Shapley value on $Q$.

Since we always assume that $Q$ is a fixed G-invariant subspace of $B V, F A \subseteq Q$, and $G$ is a group such that $H^{0}(G, F A) \neq\{0\}$, it also follows that $H^{0}(G, Q) \neq\{0\}$.

Let $A$ be any subset of $B V$, and let $v \in B V-A$. Then by $A$, we denote the R-linear subspace of $B V$ containing $A$ and $\{g v: g \in G\}$, that is,

$$
\left.A_{v}:=\operatorname{lin}_{\mathrm{R}}\{g v: g \in G\} \cup \dot{A}\right) \text {. }
$$

It is easy to see that if $A$ is the $G$-invariant subset of $B V$ then the sets $\{g v: g \in G\}$ and $A$ are disjoint, and the space $A v$ is a G-invariant subspace of $B V$.

LEMMA 3.2. Each G-Shapley value $\varphi$ on $Q$ can be extended to a $G$-Shapley value $\varphi_{v}$ defined on the $G$-invariant space $Q_{v}$, when $v \in B V-Q$.

Proof. Let $v \in B V-Q$. Then, for each $g \in G, g v \notin Q$. Indeed, if for some $g_{1} \in G, u=g_{1} v \in Q$, then $v=g_{1}^{-1} u \in Q$, because $Q$ is G-invariant, but it yields a contradiction.

Let $v=v_{1}-v_{2}$ be the Jordan decomposition of $v$. By $G_{v}$ we denote the subgroup of $G$ defined by the formula

$$
G_{v}=\{g \in G: g v=v\} \text {. }
$$

Now we extend $\varphi$ to the R-linear map $\varphi_{v}: Q_{v} \mapsto F A$ by setting 


$$
\varphi_{v}(u)= \begin{cases}\varphi(u) & \text { for } u \in Q, \\ g\left(\mu_{v}^{1}-\mu_{v}^{2}\right) & \text { for } \mu=g v, g \in G,\end{cases}
$$

where $\mu_{v}^{1}$ and $\mu_{v}^{2}$ are any elements of $F A^{+}$satisfying two conditions:

$$
\begin{gathered}
\mu_{v}^{\perp}, \mu_{v}^{2} \in H^{O}\left(G, F A^{+}\right), \\
\mu_{v}^{\perp}(X)=v_{1}(X) \text { and } \mu_{v}^{2}(X)=v_{2}(X) ;
\end{gathered}
$$

such elements exist because $H^{0}\left(G_{v}, F A^{+}\right) \supset H^{0}\left(G, F A^{+}\right) \neq\{0\}$.

It is easy to verify that the mapping $\varphi_{v}$ defined above satisfies the condition (SVI) and (SVII) on $Q_{v}$. Thus $\varphi_{v}$ is a G-Shapley value on the G-invariant space $Q_{v} \supseteq Q$.

LEMMA 3.3. A G-Shapley value $\varphi$ on the subspace $Q$ exists if and only if there exists a linear map $\varphi_{0}: Q^{G} \mapsto F A$ such that the following diagram

$(\mathrm{SVI})_{0}$

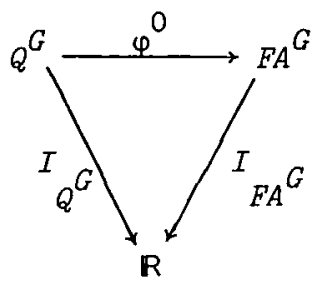

commutes and

$$
|| \circ \varphi_{0}=\varphi_{0} \circ||
$$

$\left(\varphi_{0}\right.$ is the G-Shapley value on $\left.Q^{G}\right)$.

Proof. If $\varphi$ is the $G$-Shapley value on $Q$, then $\varphi\left(Q^{G}\right) \subset F A$, and $\varphi_{0}$ defined by $\varphi_{0}=\varphi \mid Q^{G}=\varphi_{0}^{*}$ satisfies $(\mathrm{SVI})_{0}$. Indeed, it follows from Lemma 3.1, because $H^{0}(G, \mathbb{R})=\operatorname{Hom}_{\mathbb{R}[G]}(\mathbf{R}, \mathbb{R})=\mathbb{R}=\mathbf{R} \quad(G$ acts on $\mathbb{R}$ trivially). The condition (SVII) 0 is obviously satisfied. Conversely, let 
$\varphi_{0}: Q^{G} \mapsto F A^{G}$ be a R-linear map satisfying $(\mathrm{SVI})_{0}$ and (SVII) ${ }_{0}$. We shall construct the $G$-Shapley value $\varphi$ on $Q$. If $Q^{G}=Q$, then we put $\varphi=\varphi_{0}$. Now let us assume that $Q \neq Q^{G}$. Let $v \in Q-Q^{G}$. Applying Lemma 3.2 for $\varphi_{0}, Q^{G}$ and $v$ we obtain the $G$-Shapley value $\left(\varphi_{0}\right)_{v}$ on the $G$-invariant space $Q_{v}^{G} \pm Q^{G}$. Now we apply Lemma 3.2 for $\left(\varphi_{0}\right)_{v}, Q_{v}^{G}$ and $v^{\prime} \in Q-Q v_{v}^{G}$ we obtain a $G$-Shapley value $\left(\left(\varphi_{0}\right)_{v}\right)_{v^{\prime}}$ on the space $\left(Q_{v}^{G}\right)_{v^{\prime}}$, and so on. Proceeding by the transfinite induction finally we obtain a $G$-Shapley value on $Q$.

THEOREM 3.1. Let $Q$ be G-invariant subspace of BV. Assume further that $Q \supseteq F A$ and $H^{0}(G, F A)$ is nontrivial. Then there exists at least $\operatorname{dim} H^{0}(G, F A)$-distinct $G$-Shapley values on $Q$.

Proof. If $Q \supseteq F A$ and $F A^{G} \neq\{0\}$, then $Q^{G} \neq\{0\}$ and $F A$ contains the R-linear subspace isomorphic with $(R,+)$. Indeed, $F A^{G}$ is the R-linear vector space and if $v \in F A^{G}$ then $v^{+}$and $v^{-}$(in the Jordan decomposition of $v$ ) both are in $\left(F A^{+}\right)^{G}$. Since

$$
v^{+}(S)=\operatorname{supremum} \sum_{i=1}^{k} \max \left(v\left(s_{i}\right)-v\left(s_{i-1}\right), 0\right),
$$

$\emptyset=s_{0} \subset \ldots \subset s_{k}=S$ and $v^{-}=v^{+}-v$.

Thus there exists $\lambda \in\left(F A^{+}\right)^{G}$ such that $\mathbf{R} \cdot \lambda \subset F A^{G}$ and $\lambda(X)=1$. If we put

$$
\varphi_{0}(v)=v(X) \cdot \lambda \text { for all } v \in Q^{G} \text {, }
$$

then the diagram 


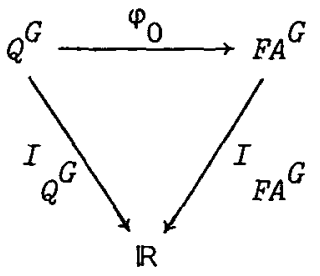

commutes .

From (3.6) we have $\varphi_{0} \circ||=|| \circ \varphi_{0}$. Hence by Lemma 3.3 there is a $G$-Shapley value on $Q$. Finally there are at least $\operatorname{dim} H^{0}(G, F A)$ many different $G$-Shapley values. For it suffices to put for every element $u$ of the basis of $H^{0}(G, F A)$ over $\mathbf{R}$,

$$
\varphi_{u}(v)=v(X) \frac{u}{u(X)}
$$

PROPOSITION 3.1. If $H^{\mathrm{O}}(G, F A)=\{0\}$ and $H^{\mathrm{O}}(G, Q) \neq\{0\}$ then there is no G-Shapley value on $Q$.

Proof. Suppose there is a G-Shapley value $\varphi$ on $Q$. Then there is $v \in H^{0}(G, Q), v(X) \neq 0$, so that, for every $g \in G$, $g \varphi(v)=\varphi(g v)=\varphi(v)$. Hence $\varphi(v) \in H^{0}(G, F A)$, that is, $\varphi(v)=0$ which is a contradiction with $\varphi(v)(X)=v(X) \neq 0$.

THEOREM 3.2. There exists a unique G-Shapley value on the space $Q$ if and only if for each $v \in Q^{+}$the space $H^{0}\left(G_{v}, F A\right)$ is one-dimensional.

REMARK. If $v \in Q^{G}$ then $G_{v}=G$ and $H^{0}\left(G_{v}, F A\right)=H^{0}(G, F A)$.

Proof. Necessity. Suppose $\operatorname{dim} H^{0}(G, F A)>1$. Let $\left\{u_{i}\right\}_{i \in I}$, $|I|>1$, be an algebraic base of the linear space $H^{0}(G, F A)$. Without loss of generality we can assume that $u_{i}(X)=1$ for every $i \in I$. Put $\varphi_{i}^{0}(v)=v(X) u_{i}$ for every $i \in I$ and $v \in Q^{G}$. obviously $\varphi_{i}^{0} \neq \varphi_{j}^{0}$ for $i \neq j, i, j \in I$.

By virtue of Lemma 3.3 we may extend each $\varphi_{u_{i}}^{0}(i \in I)$ to distinct 
Shapley values.

Similarly, suppose that $\operatorname{dim} H^{0}(G, F A)>1$ for some $v \in Q^{+}-Q^{G}$. Then there are $\mu_{1}, \mu_{2} \in H^{0}\left(G, F A^{+}\right)$such that $\mu_{1}(X)=\mu_{2}(X)=v(X)$ and $\mu_{1} \neq \mu_{2}$. We have already proved in Theorem 3.1 that there exists the $G$-Shapley value $\varphi_{0}$ over $Q^{G}$ if $H^{0}(G, F A)$ is nontrivial.

Denote by $\varphi_{1}, \varphi_{2}$ two distinct extensions of $\varphi_{0}$ over $Q_{v}$, where $\varphi_{i}(v)=v(X) \mu_{i}$ for $i=1,2$. They exist by Lemma 3.2 .

Sufficiency. Let $H^{0}\left(G_{v}, F A\right)$ be one-dimensional vector space for every $v \in Q$. Let $\varphi$ and $\psi$ be two $G$-Shapley values on $Q$. We claim that $\varphi=\psi$. Indeed, let $v \in Q^{+}$be arbitrary. It follows immediately from the definition of the G-Shapley value, that $\varphi(v), \psi(v) \in H^{0}\left(G_{v}, F A^{+}\right)$. Since $\operatorname{dim} H^{0}\left(G_{v}, F A\right)=1$, we have $\varphi(v)=c_{1} \mu, \psi(v)=c_{2} \mu$, for some $c_{1}, c_{2} \in \mathbb{R}$. Here $\mu$ is a base of the space $H^{0}\left(G_{v}, F A\right)$. But $c_{1} \mu(X)=\varphi(v)(X)=v(X)=\psi(v)(X)=c_{2} \mu(X)$. Hence $c_{1}=c_{2}$ and $\varphi(v)=\psi(v)$. Since $v$ was arbitrary then $\varphi=\psi$.

\section{References}

[1] R.J. Aumann and L.S. Shapley, Values of non-atomic games (Princeton University Press, Princeton, New Jersey, 1974).

[2] Henry Cartan and Samuel Ei lenberg, Homological algebra (Princeton University Series, 19. Princeton University Press, Princeton, New Jersey, 1956). 
[3] L.S. Shapley, "A value for n-person games", Contributions to the theory of games, Volume II, 307-317 (Annals of Mathematics Studies, 28. Princeton University Press, Princeton, New Jersey, 1953).

Institute of Mathematics,

Technical University of Wroclaw,

50-370 Wroclaw,

Wvbrzeże Wyspiańskiego 27,

Poland. 